\title{
Rare Presentations of Rapidly Growing Liposarcomas
}

\author{
Arifa Javed ${ }^{1}$, Jafar Haghshenas ${ }^{1}$, Scott Sholem ${ }^{1}$, Jamshed Zuberi ${ }^{1}$ and Farooq Shahzad ${ }^{2}$ \\ ${ }^{1}$ Department of Surgery, St. Joseph's Regional Medical Center, Paterson, NJ, USA \\ ${ }^{2}$ Division of Plastic \& Reconstructive Surgery, Ann \& Robert H Lurie Children's Hospital, Chicago, USA
}

\begin{abstract}
Liposarcomas (LSs) are a rare form of malignant tumors. They are the most common primary neoplasms of the retroperitoneum. While these tumors rarely metastasise, they are characterised by local recurrence and infiltration. Moreover, most are asymptomatic and hence grow to massive sizes before becoming symptomatic. Here, we report two unique cases of giant retroperitoneal liposarcomas seen in our urban tertiary care centre. The first case is that of a 53-year Dominican male, who developed a massive recurrence of his retroperitoneal LS within a span of just two months after initial resection- an exceedingly rare occurrence. The second case is that of a 67-year Peruvian female, who was found to have a retroperitoneal LS, causing complete displacement of the viscera in her right hemiabdomen.
\end{abstract}

Key Words: Liposarcomas, Recurrence, Retroperitoneum.

How to cite this article: Javed A, Haghshenas J, Sholem S, Zuberi J, Shahzad F. Rare Presentations of Rapidly Growing Liposarcomas. J Coll Physicians Surg Pak 2021; 31(08):982-985.

\section{INTRODUCTION}

Soft tissue sarcomas comprise $<1 \%$ of all malignant tumors with the most common variant being liposarcomas (LSs) located in the retroperitoneum. ${ }^{1}$ Retroperitoneal LSs vary in size, are usually recurrent, and due to their retroperitoneal location, grow to large sizes before getting diagnosed. ${ }^{1}$ As such, these rare forms of mesenchymal tumors, with an incidence of $2.5 / 1,000,000$ notoriously present at advanced stages and carry a poor prognosis. ${ }^{1-6}$ The majority of retroperitoneal LSs are diagnosed incidentally on computed tomography (CT) scanning. These tumors are differentiated on the basis of their histopathology with four main histological subtypes as characterised by the World Health Organization (WHO): well differentiated, dedifferentiated, myxoid, and pleomorphic. ${ }^{2}$ The mainstay of treatment involves R0 surgical resection, as LSs are insensitive to chemotherapy and local anatomy precludes effective radiation therapy (RT) in most cases. ${ }^{5}$ Here, we present two unusual cases of massive, well differentiated retroperitoneal LSs.

\section{CASE 1:}

A 53-year male with no significant medical history initially presented to the emergency department with complaints of general malaise, weakness, and persistent leukocytosis.

Correspondence to: Dr. Farooq Shahzad, Division of Plastic \& Reconstructive Surgery, Ann \& Robert H Lurie Children's Hospital, Chicago, USA

E-mail: fooqs@hotmail.com

Received: December 11, 2019; Revised: March 12, 2020;

Accepted: March 14, 2020

DOI: https://doi.org/10.29271/jcpsp.2021.08.982
A CT of abdomen and pelvis was performed which revealed a large, fatty, right retroperitoneal mass with an enhancing solid component worrisome for retroperitoneal LS. After appropriate staging workup, the patient was taken to the operating room and underwent an exploratory laparotomy with resection of the retroperitoneal mass. The mass measured $16.015 .0 \times 9.5 \mathrm{~cm}$ and pathology was consistent with well differentiated LS. Ultimately, the patient was discharged home after a short hospital course with referral to hematology/oncology for possible radiation therapy (RT).

After outpatient workup with repeat MRI, the patient was found to have a $7 \mathrm{~cm}$ right retroperitoneal mass concerning for recurrence. He, therefore, underwent a repeat triple phase CT scan of the abdomen and pelvis (Figure 1), which revealed the mass had increased in size to approximately $20 \mathrm{~cm}$ in cranio-caudal dimension. Given these findings, tumor recurrence was suspected and the patient returned to the operating room for an elective resection of this recurrent right retroperitoneal LS. The previous midline laparotomy scar was opened and upon exploration of the abdomen, there was noted to be a large right-sided retroperitoneal mass with displacement of the ascending colon and right kidney medially. The large right retroperitoneal mass was circumferentially dissected from lateral to medial (Figure 2). The mass extended down to the right psoas muscle, medially it abutted the inferior vena cava, and anteriorly the mass was closely adhered to the cecum and right colon. The retroperitoneal mass was dissected off the peritoneum, circumferentially and it was noted that posteriorly it involved some level of the fascia of the right kidney. An intraoperative urology consult was called for further evaluation, which noted that the kidney was not involved with the RPS, and therefore, the perinephric fat was left intact once the kidney was mobilized away from the mass. 


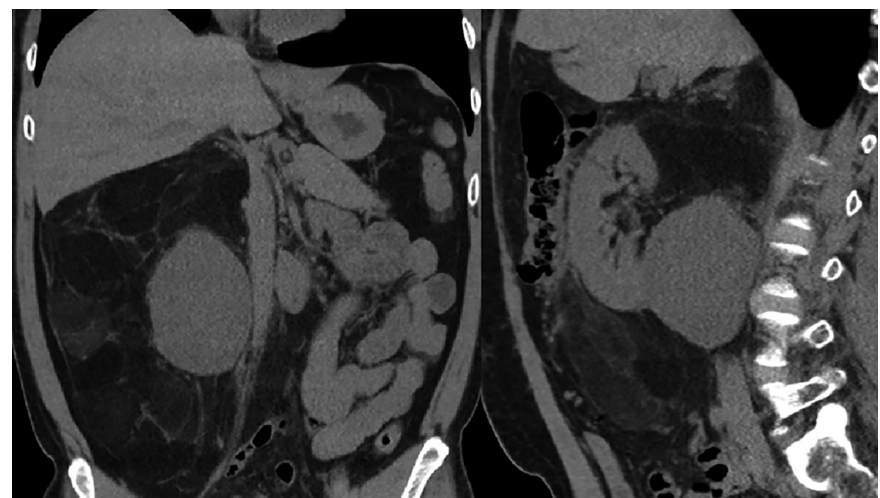

Figure 1: Abdominal CT scan demonstrating recurrent mass with solid component posterior to the right kidney. Coronal and sagittal views.

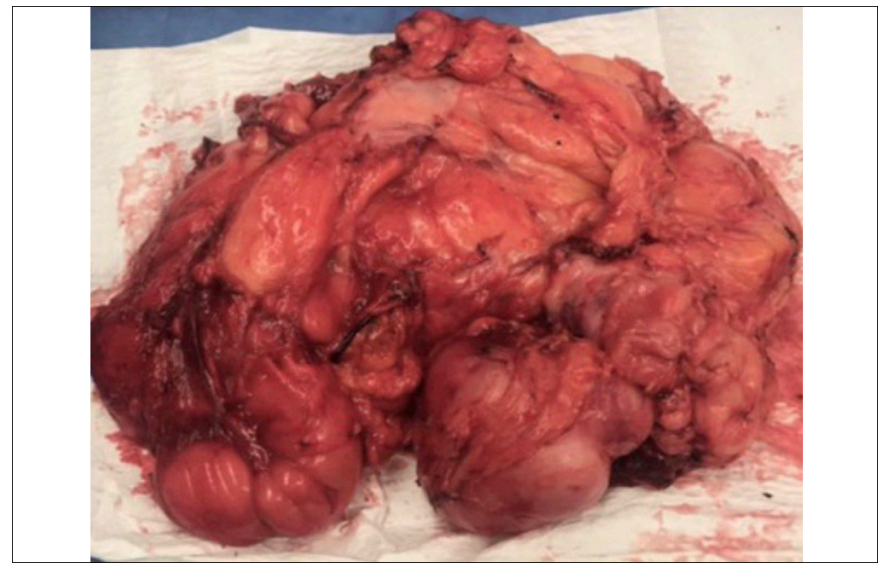

Figure 2: Recurrentright retroperitoneal mass removed en bloc.

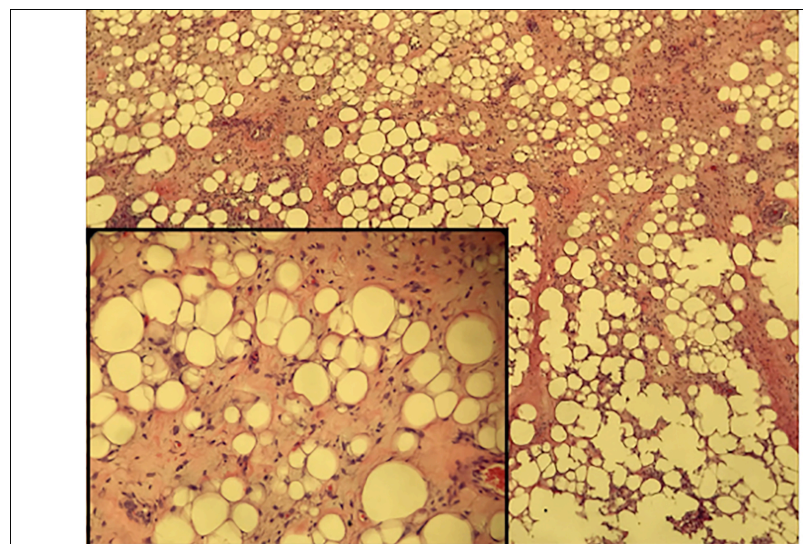

Figure 3: Lipogenic cells and many large fibrous septae. Cells contain irregularly shaped hyperchromatic nuclei.

Pathology again was consistent with well differentiated LS (Figure 3). Microscopically, the tumor demonstrated prominent variations in the size/shape of lipogenic cells and many large fibrous septae with enlarged cells containing irregularly shaped hyperchromatic nuclei. The margins, including the retroperitoneal margin had multifocal involvement by tumor with Fédération Nationale des Centres de Lutte Contre le Cancer (FNCLCC) grade I out of III, stage pT4. Following the operation, the patient was discharged home with outpatient follow-up with hematology/oncology and radiation oncology for repeat evaluation for RT given positive retroperitoneal margins on final pathology. Ultimately, after multidisciplinary evaluation, it was decided that there is no role for RT as the treatment would be too toxic given the anatomical location. Currently, the patient is being monitored with closesurveillance, with plansfor possibly repeatsurgical resection in the case of further recurrence.

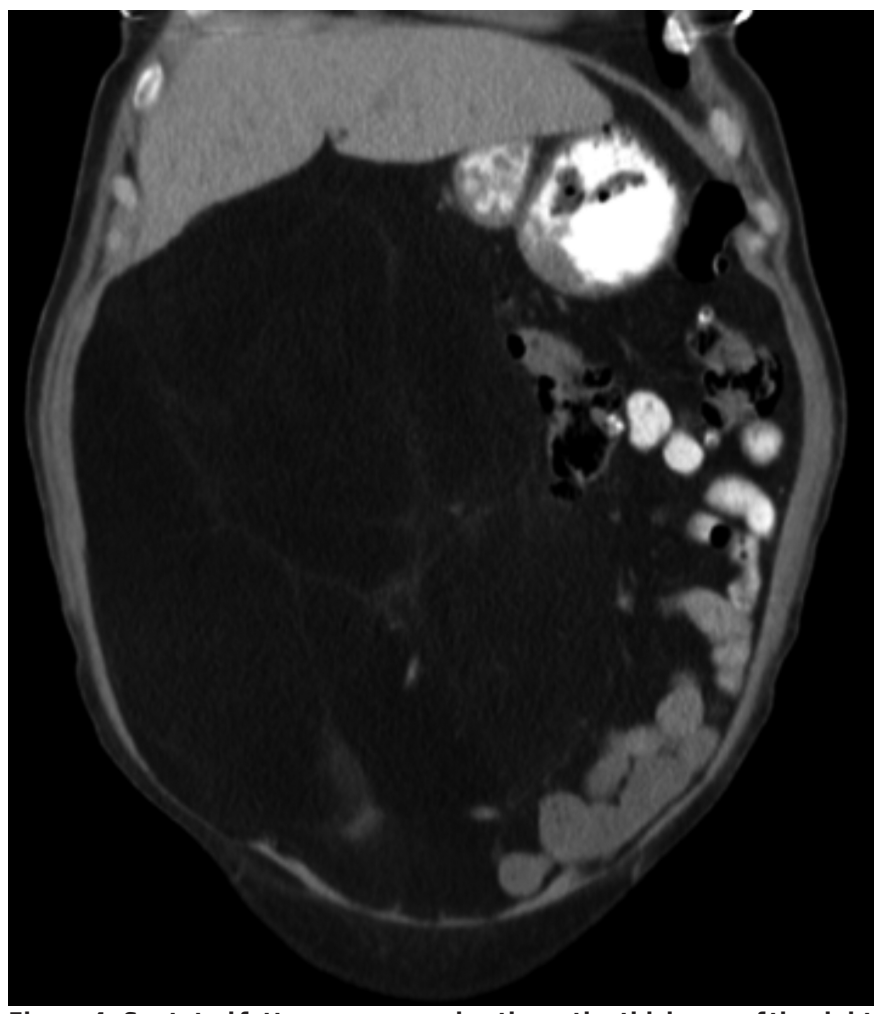

Figure 4: Septated fatty mass occupying the entire thickness of the right hemiabdomen. Note that the abdominal visceras are displaced to the left.

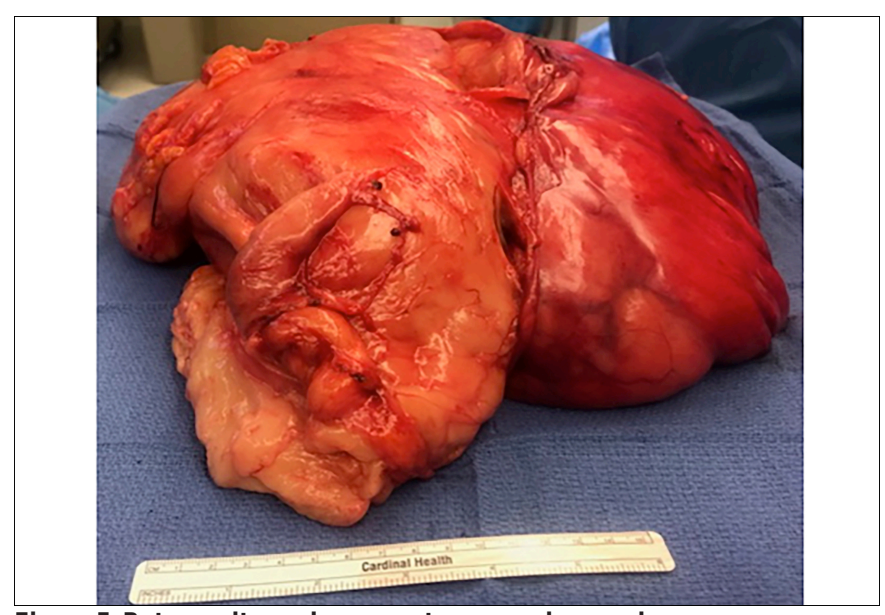

Figure 5: Retroperitoneal mass, anterosuperior specimen.

\section{CASE 2:}

A 67-year female presented to her primary care doctor after noting abdominal distention of one year duration. The distention had been accompanied by discomfort without accompanying symptoms. An abdominal CT scan and MRI both demonstrated a $30 \times 27 \times 16 \mathrm{~cm}$ septated fatty matrix mass (Figure 4). It occupied the entire thickness of the right hemiabdomen extending from the retroperitoneum to the ventral abdominal wall. 
On surgical exploration, the mass effect of LS had pushed the entire right colon across the midline. The tumor was carefully dissected from the abdominal viscera. Retroperitoneal excision involved creating a fine plane between the inferior vena cava and the tumor. The bladder and right ovary were both noted to be uninvolved by the mass. During extraction of the tumor, a cleavage line separated the mass into two components. The anterosuperior portion measured $38 \times 30 \times 11 \mathrm{~cm}$ (Figure 5), and the posteroinferior portion was $21 \times 12 \times 5 \mathrm{~cm}$. The combined total dimension of the tumor was $42 \times 36 \times 14 \mathrm{~cm}$. Intraoperative ultrasound of the liver did not show any indication of metastatic spread. The patient spent three days in the hospital and was discharged uneventfully.

Pathology returned as a well differentiated grade I LS. Microscopic examination demonstrated mature adipose tissue with focal fibrous septal formation. Within the septae, there were atypical cells showing enlarged hyperchromatic nuclei. This tumor was also pathologically staged as pT4 Nx due to multifocal involvement of the tumor margins. Follow-up will include physical examination and abdominal CT vs. MRI every 3 months for 3 years. This will be followed by annual imaging surveillance.

\section{DISCUSSION}

Retroperitoneal LSs constitute about $50 \%$ of all sarcomas. They originate from fat cells with $12-40 \%$ originating from the retroperitoneum and 35\% from the perirenal fat. ${ }^{1}$ LS have four histological subtypes: well differentiated, dedifferentiated, myxoid, and pleomorphic. Well differentiated LSs (WDLSs) have an incidence of $40-60 \%$ and are locally aggressive sarcomas with high recurrence rates despite complete surgical resection and low metastatic potential. ${ }^{2,4}$ However, when these WDLS tumors do exhibit metastasis, the lungs are by far the most common site. ${ }^{3}$ For diagnostic purposes, imaging studies like CT, or less frequently MRI, are used. CT imaging is the best modality for visualising the retroperitoneum and is very useful in estimating the amount of fat in the mass to help better classify these tumors, which is based on the amount of lipid inside the cell, the mucoid lipid, and the degree of cell differentiation., Moreover, imaging with $\mathrm{CT}$ is beneficial for ruling out any metastatic lesions in the chest, liver, or the peritoneal cavity as well as following patients with these tumors postoperatively. To make a definitive diagnosis, or in cases, where the tumor is unresectable, biopsy can be done to determine the histology of the LS. Cytogenetic studies indicate an amplification of MDM2 and CDK4 genes in well differentiated and dedifferentiated LSs. In addition, cytogenetic studies report a reciprocal translocation of $(12,16)$ (q13; p11) in myxoid/clear cell LSs; however, interestingly, no such chromosomal translocation has been isolated in WDLSs. ${ }^{3}$

In our patient, who experienced recurrence, it is unclear whether the recurrent mass originated from remnants of the LS resected two months prior or due to the natural disease progression of these types of tumors. Despite a high propensity for local recurrence, patients with LS have a relatively high 5-year survival, which is dependent on tumor histological subtype as well as tumor grade. WDLSs have an overall 5-year survival rate of $90 \%$, with the 5-year survival rate for the pleomorphic, myxoid, and dedifferentiated subtypes being 30-50\%, 60-90\%, and $75 \%$, respectively. ${ }^{1}$ Tumor grade is the most significant prognostic factor in disease prognosis for patients with LSs. ${ }^{3}$ Grading is formulated according to the Fédération Nationale des Centres de Lutte Contre le Cancer (FNCLCC), and is based on tumor differentiation/histologic subtype, location, mitotic count, and tumor necrosis. Interestingly, it is noted that lower grade of first recurrent tumor is associated with better overall survival. Therefore, in the case of both patients, having a grade I out of III (WDLSs), the 5-year survival and overall prognosis is high.

The standard of treatment for localised recurrence of retroperitoneal LSs remains repeat complete surgical resection. Despite current studies investigating the role of adjunct therapies, chemotherapy and RT are not known to have any additional benefits on the overall survival in these patients. Moreover, surgical resection provides increased survival time for patients with these tumors as seen with a case reported of a patient undergoing repeated surgical resections after continued local recurrence, which improved his survival time to 13 years. ${ }^{4}$ Given this fact, it is crucial for the treatment team to provide continued follow-up for these patients with serial imaging to identify tumor recurrence, when it happens so that patients may be directed to appropriate care with surgical resection.

LSs are a rare form of mesenchymal tumors characterised by high local recurrence and low metastatic potential. Different histologic subtypes have different recurrence rates, with the well differentiated form having comparatively lower recurrence rates. Due to high rate of local recurrence of these tumors, it is imperative to follow up these patients with routine imaging. Complete (R0) surgical resection is the standard treatment for patients with these tumors, with chemotherapy/RT having minimal benefit. Finally, in the case of local recurrence, repeat surgical resection remains the treatment of choice for these patients.

\section{CONFLICT OF INTEREST:}

The authors declared no conflict of interest.

\section{AUTHORS'CONTRIBUTION:}

AJ, JH, SS, JZ: Study design, acquisition and interpretation of data, critical revision of manuscript, and final approval.

FS: Study design, Interpretation of data, critical revision of manuscript, and final approval.

\section{REFERENCES}

1. Argadjendra M, Napitupulu R, Yudadi R, Hoetama S, Wibowo HS. Kidney sparing giant retroperitoneal liposarcoma: Case report and literature review. Int J Surg Case Rep 2019; 56:70-3. doi: 10.1016/j.ijscr.2019.02.008

2. Bagaria SP, Gabriel E, Mann GN. Multiply recurrent retroperitoneal liposarcoma. J Surg Oncol 2018; 117(1): 62-8. doi: 10.1002/jso.24929.

3. Karadayi K, Yildiz C, Karakus S, Kurt A, Bozkurt B, Soylu S, 
et al. Well-differentiated abdominal liposarcoma: Experience of a tertiary care center. World J Surg Oncol 2015; 13:166. doi: 10.1186/s12957-015-0580-z.

4. Kim EY, Kim SJ, Choi D, Lee SJ, Kim SH, Lim HK, et al. Recurrence of retroperitoneal liposarcoma: Imaging findings and growth rates at follow-up CT. AJR Am J Roentgenol 2008; 191(6):1841-6. doi: 10.2214/AJR.07.
3746.

5. Lu L, Shi HZ, Xiao ZJ, Wang D, Li CL. Clinical experience in diagnosis and treatment of primary retroperitoneal liposarcoma. Zhonghua Yi Xue Za Zhi 2018; 98(10):773-6. doi: 10.3760/cma.j.issn.0376-2491.2018.10.012.

6. Vijay A, Ram L. Retroperitoneal liposarcoma: A comprehensive review. Am J Clin Oncol 2015; 38(2):213-9. doi: 10.1097/COC.0b013e31829b5667. 